\title{
Acceptability of alcohol-free dance in place of traditional alcohol-focused events
}

Kyle Brown ${ }^{a}$, Kimberley M Hill ${ }^{b}$, Joanne Smith ${ }^{c}$, Mattias Johansson ${ }^{d}$ and Emma Davies ${ }^{e}$

aDepartment of Psychology, Faculty of Business, Law and Social Sciences, University of Birmingham, UK

${ }^{\text {b}}$ Department of Psychology, The University of Northampton, UK

'Faculty of Health and Life Sciences, University of Northumbria, Newcastle Upon Tyne, UK

'School of Health Sciences, Orebro Universitet, Sewden

eDepartment of Psychology, Oxford Brookes University, UK

\begin{abstract}
Objective: Alcohol misuse prevention often fails to account for or replace the pleasurable benefits of drinking such as relaxing and socialising with friends. Increasingly, alcohol free dance music events are emerging, allowing people to gain the positive outcomes of dancing without recourse to alcohol. This study sought to explore whether conscious-clubbing would be rated as an acceptable alternative to traditional alcohol-focused events.
\end{abstract}

Design/Setting: An online cross-sectional survey was completed by 281 young respondents (80.4\% female; mean age $=22$ ).

Method: Health-related cognitions (attitudes, intentions), perceived acceptability towards alcohol free dance events and the extent to which these were predicted by demographics and individual differences were assessed in the survey.

Results: T-tests indicated overall positive attitudes, acceptability, support towards and intention to attend alcohol-free clubbing events regardless of drinking status, with the exception of drinkers' intentions to attend an event. Exploratory multiple regression analyses indicated that young women and individuals who had previously attended these events held more positive attitudes. These attitudes were associated with acceptability and support, but more favourable attitudes towards alcohol consumption were inversely related to acceptability. More positive attitudes, previous attendance and lower life satisfaction associated with higher intentions to attend an event.

Conclusion: Results indicate that alcohol free events may provide an alternative socialising experience, with greater potential utility for young women, non-drinkers and individuals who have previously attended these events. This is of particular importance given that recent literature highlights the need for (non-alcohol) alternatives to socialise in a growing number of individuals.

Keywords: Alcohol; Alcohol free; Social drinking; Events; Acceptability

\section{Corresponding author:}

Kyle Brown, Department of Psychology, Faculty of Business, Law and Social Sciences, University of Birmingham, B4 7BD, UK

Email: kyle.brown@bcu.ac.uk 


\section{Introduction}

Alcohol misuse is associated with the increased risk of harm for young people (Babor et al., 2010; Rehm et al., 2005). University students in particular, tend to consume alcohol at harmful levels (Kypri et al., 2005; Davoren et al., 2016). University campuses provide plentiful opportunities for socialising involving alcohol, but relatively few opportunities to do so without drinking (Davies et al., 2018; Supski et al., 2017). Prevalent cultures of heavy drinking and the ubiquity of alcohol in social occasions, mean that individuals who chose not to drink are often faced with few alternatives other than to provide excuses for nondrinking, or to avoid certain contexts. Non-drinkers, for example, often feel stigmatised and may adopt strategies including pretending to drink, when attending social events (Conroy and de Visser, 2014). The lack of alternate provision means that young people may 'default' to drinking (Herring et al., 2014).

This strong culture of drinking provides a challenge to those who seek to reduce alcohol related harms by targeting individual cognitions such as attitudes and intentions. Prevention programmes that warn of the harmful consequences of drinking have been shown to be largely ineffective (Foxcroft and Tsertsvadze, 2011). Young people are generally aware of the risks, but see drinking as a pleasurable part of their social lives and actively ignore health messages (Hutton, 2012). Even those with the best of intentions to limit their drinking often fail (Sheeran, 2002) and this is particularly challenging in social situations due to peer influence (Jamison and Myers, 2008).

Existing alternative socialising options for young people at university who chose not to drink, or who want to reduce their consumption, appear to focus on promoting participation in other activities such as sports. However, team sport participation at university is typically associated with heavy drinking (Zhou et al., 2015) and one intervention that aimed to encourage student drinkers to cut down by increasing time spent exercising had no effects on consumption (Weinstock et al., 2014). One study from the USA examined the effectiveness of a programme of late night alcohol free events at a college, such as films and board games (Patrick et al., 2010). Researchers asked students to complete drinking diaries and it was found they drank less on days they attended the events, compared to normal weekend days. A more recent follow up (Layland et al., 2018) demonstrated decreased consumption and binge drinking for individuals who attended more frequently, albeit strongly driven by its impact on consumption in underage students ( $<21$ years). Similarly, Wei et al. (2010) found lower consumption and intoxication for students attending alcohol free parties. Whilst research has highlighted that a range of alcohol free alternatives are enjoyable to students, alcohol-related activities are rated as more enjoyable overall and enjoyment increased with drinking quantity (Murphy et al., 2006). Relatedly, alcohol has been reported to result in social benefits for students (Nyström, 1992) and increase bonding, particularly in the very early stages of their course (de Visser et al., 2013; Jacobs et al., 2018). One activity not examined by (Murphy et al., 2006) was live music events. Recent studies with young people suggest they are seeking alternative, credible non-drinking experiences, where they do not feel pressured to drink (Davies et al., 2018). Alcohol free live music events may provide such experiences, given the evidence underscoring the importance of music in young people's lives for identity, social and emotional development (Miranda, 2013).

Live music events have demonstrated an ability to connect people, promoting a sense of community, cooperation and altruism (Launay et al., 2016; Reddish et al., 2013). 
During these events, social structures are temporarily disbanded, allowing for the expression of other identities and a merging into oneness (Salamone, 2004) with some forms of music creating experiences of transcendence and flow (Goulding et al., 2002; Johner, 2015). Alcohol-free live music events are becoming more commonplace. One example of this is Morning Gloryville, which began in London in 2013 and claims to be the first to bring 'conscious clubbing' to the masses (Morning Gloryville, 2016). At these "sober raves", all the elements of traditional clubbing are present, other than alcohol and other drugs. This reflects the traditional conceptualisation of raves, a cultural movement conceived in response to public disapproval for excess alcohol consumption (Measham and Brain, 2005) during the late 1980s and early 1990s (Anderson and Kavanaugh, 2007). Many participated to gain a sense of community without the negative consequences of alcohol (Goulding et al., 2002).

Young people's alcohol consumption and binge drinking in the UK (for both 16-24 and 25-44 year olds) is decreasing (ONS, 2017). Sober raves may offer an alcohol free alternative that promotes social bonding, which is of particular importance to university students. We therefore sought to examine the potential of alcohol-free music events as an intervention strategy to reduce alcohol consumption by determining their acceptability as an alternative to traditional alcohol focused events.

\section{Aims}

This study had two aims: 1 . to explore health-related cognition (attitudes, intentions) towards and perceived acceptability of introducing, alcohol free dance events and whether these factors were influenced by drinking status; and 2. to explore the extent to which demographic factors, alcohol consumption, social connectedness and life satisfaction predict the aforementioned health cognitions and perceived acceptability ratings

\section{Materials and Methods}

\section{Design \& Procedure}

A cross-sectional survey design was implemented. Participants were incentivised to take part with a prize draw to win an iPad.

UK university participants were invited through research participation schemes at universities associated with the authors of this paper (in exchange for course credit, independent of the prize draw), with all other participants invited via a link distributed via social media.

Measures were presented in Qualtrics Survey Software and took approximately 15 minutes to complete.

\section{Participants}

A convenience sample of 346 respondents above 18 years old was initially recruited.

\section{Measures}

Demographics 
Age, Gender, Ethnicity and Socio-Economic Position (using education level and postcode), with the latter being used to derive the relative levels of deprivation in English neighbourhoods (Oguz et al., 2013; McLennan et al., 2011).

\section{Alcohol Consumption}

The Alcohol Use Disorders Identification Test (Babor et al., 2001) was used as a self-report indicator of potentially hazardous consumption levels. The AUDIT is a nationally validated, ten-item screening measure often employed in research and pre-clinical settings (e.g. Primary care, Accident and Emergency) to indicate problematic levels of alcohol consumption. Example questions include: "How often do you have a drink containing alcohol?". Participants were asked to respond to questions on either 3 or 5-point likert scales. Individuals scoring 0 on the AUDIT (indicating no consumption) were also asked: "Roughly how long have you abstained from alcohol in months?" and "What are your reasons for abstaining from alcohol?" A Cronbach's alpha value of .81 indicated very good internal consistency within the sample.

\section{Acceptability}

Acceptability indices were adapted from (Petrescu et al., 2016), who assessed the public acceptability of government "nudge" interventions aimed to reduce consumption of sugary drinks. Participants were first shown a video clip highlighting a sober rave (https://www.youtube.com/watch?v=OeMScv8er5Y). The following scenarios were presented for university students and (non-students) respectively: "Your university (employer) is going to introduce an alcohol-free social event, like the one in the video, in fresher's week (for employees to attend), instead of a traditional club night involving alcohol consumption." Acceptability was then assessed on a 7-point (1-7) scale, using the following two items: "Do you support or oppose this policy?" and "How acceptable to you find this policy?". Internal consistency of these responses was good $(\alpha=.74)$.

\section{Drinking-related health cognition}

Health cognitions derived from the theory of planned behaviour (Ajzen, 2005) were assessed using 7-point semantic differential scales, according to (Norman, 2011). Attitudes to alcohol consumption were assessed with the statement "Engaging in alcohol consumption next month will be..." and the anchors "Bad - Good; Foolish - Wise; Unpleasant - Pleasant; Enjoyable - Unenjoyable. (Definitely do not intend to - Definitely intend to)". Internal consistency was high $(\alpha=.90)$. Other health cognitions were assessed using 7-point Likert scales ranging from strongly disagree to agree. Intention to consume alcohol was assessed with the following: "To what extent do you intend to engage in alcohol consumption over the next month? and Intentions to attend an event were assessed with "If there was an event near me, like the one shown in the video, in the next four weeks then I would intend to go along". "People who are important to me think I should engage in alcohol consumption over the next month" and "I feel in complete control over whether or not I engage in alcohol consumption over the next month" provided indices for subjective norm and perceived behavioural control respectively. 
Attitudes towards alcohol free dance events were assessed using 7-point semantic differentials, based on previous studies of attitudes towards consumption of alcohol free drinks (van der Zwaluw et al., 2013) and binge drinking (Norman, 2011), with the following phrase: "I think alcohol-free events like these seem..." and the same anchors were used, yielding a high internal consistency $(\alpha=.85)$.

\section{Predictors of Engagement with Alcohol-free events}

Social connectedness: The revised Social connectedness questionnaire (Lee and Robbins, 1995) was used to assess the degree to which individuals felt connected to others in their social environment. This 8-item scale required responses on a 5-point likert scale. Example items included: "I feel disconnected to the world around me" and "Even among my friends, there is no sense of brother/sisterhood". Internal consistency was excellent ( $\alpha=.96)$.

Satisfaction with Life: The Satisfaction with Life Scale (Diener et al., 1985) was used to assess cognitive judgement regarding satisfaction with one's life. This 5 -item scale required participants to respond on a 7-point likert scale, ranging from 1 (Strongly Disagree) to 7 (Strongly Agree), with items including: "In most ways my life is close to ideal" and "So far I have gotten the important things I want in life". Reliability analyses also demonstrated very good internal consistency $(\alpha=.88)$

\section{Statistical Analyses}

IBM SPSS statistics (version 24: IBM, 2016) was used to determine the overall acceptability (using acceptability and support indices), attitudes towards and intentions to attend alcohol free dance events. Based on the 7-point scales, with larger values indicating an increase for each of these indices, one sample t-tests (with a midpoint of 4 indicating ambivalent responses) were conducted to indicate whether responses significantly deviated from this scale midpoint. These analyses were conducted both overall and split according to selfreported drinking status. Ratings were then directly compared according to drinking status (using independent t-tests). Regression analyses were then conducted to determine the relative extent to which demographic variables (age, gender), problematic alcohol consumption (AUDIT scores), health cognitions (attitudes, subjective norm, perceived behavioural control), social connectedness, life satisfaction and past behaviour (previous attendance) predicted each of the four aforementioned indices.

\section{Results}

Of the 346 initial respondents, a total of 281 completed the questionnaire. The majority of these individuals were female $(80.4 \%)$, students $(91.1 \%)$ drinkers based on audit self-report $(85.8 \%)$ of white European ethnicity $(70.5 \%)$ with an average age of 22 years (SD $=7.65$ ). Sixty (21.4\%) of the questionnaire completers had previously attended an alcohol-free event. These individuals were typically female $(N=46)$ and older $(M=26.07 \vee 20.83)$, with lower AUDIT scores ( $M=7.05 \vee 8.22)$, greater social connectedness $(M=3.84 \vee 3.68)$ and satisfaction with life ( $M=22.46$ v 21.15) when compared with non-attenders. (See OLSM 1 for a breakdown) 


\section{Overall ratings}

A series of one sample t-tests (table 1 ) indicated that the overall sample displayed positive attitudes, acceptability and support for the events. However, people did not intend to attend the events.

[Please insert table 1 here.]

Ratings split according to drinking status were largely consistent with overall ratings (see OLSM 2), with the exception of non-drinker intentions, which were ambivalent (not significantly different from the scale midpoint), $t(39)=.838, p=.41, d=.13$. This indicates that the overall (negative) intentions to attend was largely driven by individuals that consume alcohol.

\section{Ratings according to drinking status}

Independent sample t-tests indicated that there was a significant difference between drinkers and non-drinkers with regards to all outcomes, with non-drinkers responding higher for each metric.

[Please insert table 2 here.]

\section{Predictors of health cognitions related to and acceptability for alcohol free dance events}

Attitudes towards the events

Linear regression analyses demonstrated that (Female) gender, older age, previous attendance and lower intentions to consume alcohol were all positively related to increased event attitudes (see Table 3).

[Please insert table 3 here.]

Intention to attend an event

Previous attendance, higher event attitudes, higher alcohol-related subjective norms and lower life satisfaction were associated with increased intention to attend an event (see Table 4).

[Please insert table 4 here.]

Acceptability

(Positive) event attitudes and negative attitudes towards alcohol consumption were significantly related to increased acceptability (see Table 5).

[Please insert table 5 here.] 


\section{Support}

(Female) gender, increased event attitudes and lower social connectedness were all associated with increased support for events (see Table 6).

[Please insert table 6 here.]

\section{Discussion}

This study investigated the extent to which alcohol free dance events could serve as a viable alternative to traditional alcohol focused events. It involved and examination of healthrelated cognitions and acceptability in a predominantly young, female white (European) student sample. These individuals were found to be generally supportive of these events as an alternative to traditional alcohol-focused events within their university or place of work, as indexed by positive attitudes, acceptability and support ratings that were more pronounced in non-drinkers. Intentions to attend an event were more mixed, however, with drinkers and non-drinkers providing negative and ambivalent ratings respectively.

\section{Overall acceptability and support}

The overall acceptability and support for implementation of alcohol free dance events (regardless of drinking status) could reflect a general decline in alcohol consumption for young people across European countries (Kraus and Nociar, 2016) and throughout the world (Pennay et al., 2018). Such declines are considered to be largely driven by young people in contrast to other age brackets (Livingston and Dietze, 2016). Some argue that increased concern for acute harms (e.g. injuries), knowledge of health-related risks and increased parental restrictions (Livingston and Callinan, 2017) have contributed to this.

A growing body of research has begun to focus on lifetime alcohol abstinent individuals reaching college age (Rinker and Neighbors, 2013) with a US national survey indicating that approximately $20 \%$ of people are abstinent (Johnston et al., 2011). This literature provides useful insights into the reasons for not consuming alcohol. Reasons for not consuming alcohol in such samples include personal values (such as religion), no interest, peer disapproval, health concerns and wasting money (Bernards et al., 2009). Of interest, Rinker and Neighbors (2013) highlight the increasing evidence for currently abstinent (i.e. < 3 months) individuals progressing to heavy episodic drinking in college (Baer et al., 1995; McCabe et al., 2005). They suggested that in order to maintain abstinence, individuals were required to resist conformity from their peers and/or change their support network. Others also argue we should focus more on drinking contexts and practices as opposed to solely individual differences (Meier et al., 2018; Supski et al., 2017). Additionally, Freshers' week in particular can result in exclusion for alcohol abstinent individuals (Jacobs et al., 2018). Together, this highlights how there can be a discrepancy between (positive) acceptability towards alternatives, alongside low intentions to undertake the behavior. Similarly, $81 \%$ of a nationally representative cross-sectional survey of 972 UK participants (even more so the younger people) indicated no intentions to reduce their drinking (Rosenberg et al., 2017). It can thus be argued that changing social norms and drinking contexts (i.e. getting people to experience these events) could potentially influence intentions to engage with non-drinking social practices. 


\section{Predictors of primary outcomes}

The secondary aim of the study was to determine the impact of participant characteristics on acceptability ratings. Importantly, the range of (sometimes contradictory) findings can be potentially explained by the aforementioned combination of peer pressure, alcohol-related default contexts and lack of alternatives.

\section{Predictors of acceptability and support}

Whilst attitudes towards alcohol free dance events were positively associated with acceptability and support, attitudes towards alcohol consumption were inversely related to acceptability and not related to support. Previous attendance, (female) gender and lower social connectedness resulted in greater support, but not acceptability for alternative events. The association between increased event attitudes and their acceptability/support as a substitute for alcohol focused events aligns with evidence for a decline in alcohol consumption, specifically in young people (Pennay et al., 2018; Livingston and Dietze, 2016). It could also be speculated that this sample of predominantly young university students includes initially abstinent individuals (Johnston et al., 2011) and/or those feeling pressured to default to drinking during their university experience (Herring, Bayley, \& Hurcombe, 2014; Jacobs, Conroy \& Parke, 2018). In turn, this potentially explains the negative relationship between attitudes towards alcohol consumption and acceptability; individuals with less favourable attitudes may have been already abstinent, pressured or more aware of the negative consequences and thus potentially more accepting of an alternative.

Individuals who had previously attended an event were perhaps more supportive (and had more favourable attitudes and intentions) based on previous positive experience, or a mere exposure effect (Zajonc, 1968) at the very least. One might speculate the discrepancy between acceptability and support in this instance is derived from individuals' desire for an additional way to express themselves, but perhaps not at the expense of another (i.e. raving with alcohol). In support, many people enjoy raves because they allow people to express themselves freely (Goulding et al., 2002) and that they can play a role in the formation of emerging identities (Miranda, 2013). Similarly, fostering identity and freedom of expression could be an incentive for individuals low in social connectedness to provide greater support for alternatives, especially if they have found it difficult to gain social ties through traditional (alcohol-focused) events. Greater support for alcohol free dance events for young women relative to men could be attributable to greater prevalence lifetime abstinence in women (Choi et al., 2016) and/or evidence suggesting that college age males (compared to females) tend to consume alcohol for social and enhancement reasons (Kuntsche et al., 2006).

\section{Predictors of attitudes towards alcohol free dance events}

Somewhat consistent with acceptability and support outcomes, female gender and previous attendance were positively associated with attitudes towards the events themselves. We posit that a combination of greater abstinence, different consumption motivations and/or mere exposure could also potentially explain these findings. In addition, older age and lower intentions to consume alcohol were also positively associated with event attitudes. As with 
the inverse association between alcohol-related attitudes and acceptability, we speculate that those with lower alcohol-consumption intentions may like the idea of avoiding both the pressure to consume alcohol and the negative consequences it entails, whilst gaining an additional opportunity for social connectedness. When considering (older) age, it must be noted that the sample predominantly consisted of relatively young university students with a mean age of 22. Further research inquiry is needed to determine the potential reasons for this.

\section{Predictors of intention to attend an event}

Attitudes towards and previous attendance of alcohol free dance events were also associated with higher intentions to attend an event. These have been addressed above. In addition, positive alcohol-related subjective norms and lower life satisfaction were associated with increased intentions to attend. Phrasing of the subjective norm item "People who are important to me think I should engage in alcohol consumption over the next month" could provide insight into the somewhat paradoxical association between positive alcohol-related subjective norms and intentions, with such individuals perhaps feeling pressured to socialise in alcohol-related contexts in order to maintain friendships (Herring et al., 2014).

\section{Limitations}

We acknowledge that a number of insights outlined in the discussion are speculatory and urge further research to understand why certain traits are associated with certain perceptions of alcohol free dance events. Specifically, we posit that sober-raves may provide an alcohol-free setting within which students can bond that differs from traditional (alcoholrelated) bonding (de Visser et al., 2013), but have not explicitly tested this.

Findings are also somewhat limited by the specific portrayal; a video version of a specific (morning) event, including predominantly women of an age older than the present sample is perhaps too much of a departure from a traditional dance event. Not only could individuals' personal experiences differ from this, but there are also other non-alcohol focused events that promote consciousness altering through other means (e.g. taking drugs). Whilst this particular video clip was selected based on the popularity of the event that was portrayed, its generalisability requires consideration for the feasibility of such alcohol-free interventions for all. Finally, the convenience sample of young women somewhat limits the generalisability of the findings.

\section{Implications}

Our findings provide some support for the potential of alcohol-free live music events as a credible experience. Specifically, introducing such events early on in the university experience (i.e. during Freshers' week) might provide a friendly experience that naturally increases social bonding especially for those low in social connectedness and life satisfaction. Non-drinking students would potentially benefit from such an intervention, preventing them from defaulting to alcohol consumption in order to gain friendships. As such, we would argue that the former have particular potential for aiding in the transition to university. This study has provided insights regarding the feasibility that sober rave-naive 
individuals might decide to try attending an event. It is important to note that certain types of alcohol-free events may not appeal to all demographics (particularly young men) however and more research is required to determine how they might view such events more favourably. This could be achieved through more detailed examination of the reasons why people do (and others do not) choose to attend such a particular kind of event and, as expressed above, ascertain how people experience these events for the first time.

\section{Funding}

Oxford Brookes University provided funding for an iPad Mini as a part of a prize draw for participants who took part in the study.

\section{Conflict of interest}

There are no conflicts of interest to report for any of the authors of this manuscript. Ethical approval was provided from the Faculty of Business, Law \& Social Sciences Ethics Committee within Birmingham City University, April 2017: 076/17)

\section{Acknowledgements}




\section{References}

Ajzen I. (2005) Attitudes, personality, and behavior Maidenhead: McGraw-Hill Education (UK).

Anderson TL and Kavanaugh PR. (2007) A 'rave'review: conceptual interests and analytical shifts in research on rave culture. Sociology Compass 1: 499-519.

Babor T, Caetano R, Casswell S, et al. (2010) Alcohol No Ordinary Commodity - Research and public policy. Oxford, UK: Oxford University Press.

Babor TF, Higgins-Biddle JC, Saunders JB, et al. (2001) Audit. The Alcohol Use Disorders Identification Test (AUDIT): guidelines for use in primary care. Geneva: World Health Organisation.

Baer JS, Kivlahan DR and Marlatt GA. (1995) High-risk drinking across the transition from high school to college. Alcoholism: Clinical and experimental research 19: 54-58.

Bernards S, Graham K, Kuendig H, et al. (2009) 'I have no interest in drinking' : a crossnational comparison of reasons why men and women abstain from alcohol use. Addiction 104: 1658-1668.

Choi NG, DiNitto DM, Marti CN, et al. (2016) Sociodemographic characteristics and health status of lifetime abstainers, ex-drinkers, bingers, and nonbingers among baby boomers and older adults. Substance Use \& Misuse 51: 637-648.

Conroy D and de Visser R. (2014) Being a non-drinking student: An interpretative phenomenological analysis. Psychology \& Health 29: 536-551.

Davies EL, Law C and Hennelly S. (2018) 'You have to be prepared to drink'. Young adults' reflections on changing social drinking behaviours at university. Health Education 118(1): 2-16.

Davoren MP, Demant J, Shiely F, et al. (2016) Alcohol consumption among university students in Ireland and the United Kingdom from 2002 to 2014: a systematic review. Bmc Public Health 16: 1-13.

de Visser RO, Wheeler Z, Abraham C, et al. (2013) 'Drinking is our modern way of bonding': Young people's beliefs about interventions to encourage moderate drinking. Psychology \& Health 28: 1460-1480.

Diener ED, Emmons RA, Larsen RJ, et al. (1985) The satisfaction with life scale. Journal of Personality Assessment 49: 71-75.

Foxcroft DR and Tsertsvadze A. (2011) Universal school-based prevention programs for alcohol misuse in young people. Cochrane Database of Systematic Reviews 9: 1-54.

Goulding C, Shankar A and Elliott R. (2002) Working Weeks, Rave Weekends: Identity Fragmentation and the Emergence of New Communities. Consumption Markets \& Culture 5: 261-284.

Herring R, Bayley M and Hurcombe R. (2014) "But no one told me it's okay to not drink": a qualitative study of young people who drink little or no alcohol. Journal of Substance Use 19: 95-102.

Huang J-H, DeJong W, Schneider SK, et al. (2011) Endorsed reasons for not drinking alcohol: a comparison of college student drinkers and abstainers. Journal of Behavioral Medicine 34: 64-73.

Hutton F. (2012) Harm reduction, students and pleasure: An examination of student responses to a binge drinking campaign. International Journal of Drug Policy 23: 229235. 
Jacobs L, Conroy D and Parke A. (2018) Negative experiences of non-drinking college students in Great Britain: an interpretative phenomenological analysis. International Journal Of Mental Health and Addiction 16: 737-750.

Jamison J and Myers LB. (2008) Peer-group and price influence students drinking along with planned behaviour. Alcohol and Alcoholism 43: 492-497.

Johner A. (2015) Transformational Festivals: A New Religious Movement? . In: Simao E (ed) Exploring Psychedelic Trance and Electronic Dance Music in Modern Culture. Hershey, PA, USA: IGI Global, 58-86.

Johnston LD, O'Malley PM, Bachman JG, et al. (2011) Monitoring the Future national survey results on drug use, 1975-2010. Volume I: Secondary school students. Ann Arbor: Institute for Social Research, The University of Michigan.

Kraus L and Nociar A. (2016) ESPAD report 2015: results from the European school survey project on alcohol and other drugs. Luxembourg: European Monitoring Centre for Drugs and Drug Addiction.

Kuntsche E, Knibbe R, Gmel G, et al. (2006) Replication and validation of the Drinking Motive Questionnaire Revised (DMQ-R, Cooper, 1994) among adolescents in Switzerland. European Addiction Research 12: 161-168.

Kypri K, Cronin M and Wright CS. (2005) Do university students drink more hazardously than their non-student peers? Addiction 100: 713-714.

Launay J, Tarr B and Dunbar RIM. (2016) Synchrony as an Adaptive Mechanism for LargeScale Human Social Bonding. Ethology 122: 779-789.

Layland EK, Calhoun BH, Russell MA, et al. (2018) Is Alcohol and Other Substance Use Reduced When College Students Attend Alcohol-Free Programs? Evidence from a Measurement Burst Design Before and After Legal Drinking Age. Prevention Science20: 342-352.

Lee RM and Robbins SB. (1995) Measuring belongingness: The social connectedness and the social assurance scales. Journal of Counseling Psychology 42: 232.

Lennox JC and Forsyth AJM. (2015) Assessing the role that entertainers play in alcohol marketing and the maintenance of good order within on-trade licensed premises. London: Alcohol Research UK.

Livingston M and Callinan S. (2017) Changing attitudes to alcohol in Australia. Journal of Studies on Alcohol and Drugs 78: 844-852.

Livingston M and Dietze P. (2016) National survey data can be used to measure trends in population alcohol consumption in Australia. Australian and New Zealand Journal of Public Health 40: 233-235.

McCabe SE, Hughes TL, Bostwick W, et al. (2005) Assessment of difference in dimensions of sexual orientation: implications for substance use research in a college-age population. Journal of Studies on Alcohol 66: 620-629.

McLennan D, Barnes H, Noble M, et al. (2011) The English indices of deprivation 2010. London: Department for Communities and Local Government.

Measham F and Brain K. (2005) 'Binge'drinking, British alcohol policy and the new culture of intoxication. Crime, Media, Culture 1: 262-283.

Meier PS, Warde A and Holmes J. (2018) All drinking is not equal: how a social practice theory lens could enhance public health research on alcohol and other health behaviours. Addiction 113: 206-213.

Miranda D. (2013) The role of music in adolescent development: much more than the same old song. International Journal of Adolescence and Youth 18: 5-22. 
Morning Gloryville. (2016) About us. Available at: http://morninggloryville.com/about-us/. Murphy JG, Barnett NP and Colby SM. (2006) Alcohol-related and alcohol-free activity participation and enjoyment among college students: A behavioral theories of choice analysis. Experimental and Clinical Psychopharmacology 14: 339.

Norman P. (2011) The theory of planned behavior and binge drinking among undergraduate students: Assessing the impact of habit strength. Addictive Behaviors 36: 502-507.

Nyström M. (1992) Positive and negative consequences of alcohol drinking among young university students in Finland. British Journal of Addiction 87: 715-722.

Oguz S, Merad S and Snape D. (2013) Measuring National Well-being-What matters most to Personal Well-being? Newport: Office of National Statistics.

ONS. (2017) Adult drinking habits in Great Britain 2005-2016. Newport: Office of National Statistics.

Patrick ME, Maggs JL and Osgood DW. (2010) LateNight Penn State Alcohol-Free Programming: Students Drink Less on Days They Participate. Prevention Science 11: 155-162.

Pennay A, Holmes J, Törrönen J, et al. (2018) Researching the decline in adolescent drinking: The need for a global and generational approach. Drug and Alcohol Review 37: S115S119.

Petrescu DC, Hollands GJ, Couturier D-L, et al. (2016) Public acceptability in the UK and USA of nudging to reduce obesity: the example of reducing sugar-sweetened beverages consumption. PLoS One 11: e0155995.

Reddish P, Fischer R and Bulbulia J. (2013) Let's Dance Together: Synchrony, Shared Intentionality and Cooperation. Plos One 8: e71182.

Rehm J, Room R, van den Brink W, et al. (2005) Alcohol use disorders in EU countries and Norway: An overview of the epidemiology. European Neuropsychopharmacology 15: 377-388.

Rinker DV and Neighbors C. (2013) Reasons for not drinking and perceived injunctive norms as predictors of alcohol abstinence among college students. Addictive Behaviors 38: 2261-2266.

Rosenberg G, Bauld L, Hooper L, et al. (2017) New national alcohol guidelines in the UK: public awareness, understanding and behavioural intentions. Journal of Public Health Vol(issue): $1-8$.

Salamone FA. (2004) Encyclopedia of Religious Rites, Rituals and Festival. New York: Routledge.

Sheeran P. (2002) Intention-behavior relations: A conceptual and empirical review. In: Hewstone M and Stroebe W (eds) European Review of Social Psychology. Chichester, UK: John Wiley \& Sons, 1-36.

Supski S, Lindsay J and Tanner C. (2017) University students' drinking as a social practice and the challenge for public health. Critical Public Health 27: 228-237.

van der Zwaluw CS, Kleinjan M, Lemmers L, et al. (2013) Longitudinal associations between attitudes towards binge drinking and alcohol-free drinks, and binge drinking behavior in adolescence. Addictive behaviors 38: 2110-2114 \%@ 0306-4603.

Wei, J., Barnett, N.P. and Clark, M. (2010) Attendance at alcohol-free and alcohol-service parties and alcohol consumption among college students. Addictive Behaviors 35(6): 572-579. 
Weinstock J, Capizzi J, Weber SM, et al. (2014) Exercise as an intervention for sedentary hazardous drinking college students: A pilot study. Mental Health and Physical Activity 7: 55-62.

Zajonc RB. (1968) Attitudinal effects of mere exposure. Journal of Personality and Social Psychology 9: 1.

Zhou J, Heim D and O'Brien K. (2015) Alcohol Consumption, Athlete Identity, and Happiness Among Student Sportspeople as a Function of Sport-Type. Alcohol and Alcoholism 50: 617-623. 
Table 1: Means and inferential statistics for the one sample t-tests on attitudes, acceptability, support and intention to attend events (with a scale midpoint of 4).

\begin{tabular}{rcccccc} 
& $x$ & $S D$ & $t$ & $d f$ & $p$ & $d$ \\
\hline Alcohol-free Event Attitudes & 4.56 & 1.49 & 6.26 & 278 & $<.001^{* *}$ & 0.38 \\
Acceptability & 5.52 & 1.61 & 15.80 & 278 & $<.001^{* *}$ & 0.94 \\
Support & 4.64 & 1.82 & 5.64 & 258 & $<.001^{* *}$ & 0.35 \\
Intention & 3.28 & 1.83 & -6.60 & 278 & $<.001^{* *}$ & -0.39 \\
\hline
\end{tabular}

$* \mathrm{p}<.05 \quad * * \mathrm{p}<.01$ 
Table 2: Means and inferential statistics for attitudes, acceptability, support and intention to attend events, according to drinking status.

\begin{tabular}{rcccccccc} 
& \multicolumn{1}{c}{ Non-drinker } & \multicolumn{1}{c}{ Drinker } & & & & \\
\cline { 2 - 8 } & $x$ & $S D$ & $x$ & $S D$ & $t$ & $d f$ & $p$ & $d$ \\
\hline Alcohol-free Event Attitudes & 5.28 & 1.78 & 4.44 & 1.41 & 3.36 & 277 & $.001^{* *}$ & 0.52 \\
Acceptability & 6.25 & 1.30 & 5.40 & 1.62 & 3.70 & 61.48 & $<.001^{* *}$ & 0.58 \\
Support & 5.85 & 1.54 & 4.46 & 1.80 & 4.23 & 257 & $<.001^{* *}$ & 0.83 \\
Intention & 4.28 & 2.08 & 3.11 & 1.74 & 3.82 & 277 & $<.001^{* *}$ & 0.51 \\
\hline
\end{tabular}

$* p<.05 \quad * * p<.01$ 
Table 3: Regression analysis examining impact of demographics, alcohol consumption, health cognitions, social connectedness, life satisfaction and past behaviour on event attitudes

\begin{tabular}{rccccc} 
& $B$ & $S E(B)$ & $b$ & $|t|$ & $p$ \\
\hline Gender & -.49 & .19 & -.14 & -2.58 & $.010^{* *}$ \\
Age & .05 & .01 & .24 & 3.92 & $<.001^{* *}$ \\
AUDIT & -.01 & .02 & -.02 & -.28 & .782 \\
Previous Attendance & .67 & .21 & -.19 & -3.26 & $.001^{* *}$ \\
Alcohol Consumption Attitudes & -.02 & .07 & -.03 & -.31 & .757 \\
Intention & -.12 & .06 & -.19 & -2.02 & $.045^{*}$ \\
Subjective Norm & .06 & .07 & .06 & .80 & .422 \\
Perceived behavioural control & .09 & .07 & .08 & 1.32 & .189 \\
Life Satisfaction & .02 & .01 & .09 & 1.56 & .121 \\
Social Connectedness & .06 & .07 & .05 & .82 & .416 \\
$\mathrm{R}^{2}$ & .25 & & & & \\
Adjusted $\mathrm{R}^{2}$ & .22 & & & & \\
\hline
\end{tabular}

$* \mathrm{p}<.05 \quad * * \mathrm{p}<.01$

Model fit: $F(10,272)=8.570, P<.001$ 
Table 4: Regression analysis examining impact of demographics, alcohol consumption, health cognitions, social connectedness, life satisfaction and past behaviour on intentions to attend an event

\begin{tabular}{rccccc} 
& $B$ & $S E(B)$ & $b$ & $|t|$ & $p$ \\
Gender & .02 & .17 & .00 & .11 & .912 \\
Age & -.01 & .01 & -.02 & -.44 & .660 \\
AUDIT & -.00 & .02 & -.00 & -.07 & .945 \\
Previous Attendance & .48 & .18 & -.11 & -2.60 & $.010^{* *}$ \\
Alcohol-free event attitudes & .91 & .05 & .74 & 16.91 & $<.001^{* *}$ \\
Alcohol consumption Attitude & -.15 & .06 & -.16 & -2.53 & $.012^{*}$ \\
Intention & .028 & .05 & .03 & .51 & .608 \\
Subjective Norm & .17 & .06 & .14 & 2.75 & $.006^{* *}$ \\
Perceived behavioural control & -.08 & .06 & -.06 & -1.31 & .190 \\
Life Satisfaction & -.03 & .01 & -.12 & -2.86 & $.005^{* *}$ \\
Social Connectedness & .06 & .06 & .04 & 1.00 & .318 \\
$\mathrm{R}^{2}$ & .62 & & & & \\
Adjusted $\mathrm{R}^{2}$ & .60 & & & & \\
\hline & & & & &
\end{tabular}

$* \mathrm{p}<.05 \quad * * \mathrm{p}<.01$

Model fit: $F(11,272)=38.514, p<.001$ 
Table 5: Regression analysis examining impact of demographics, alcohol consumption, health cognitions, social connectedness, life satisfaction and past behaviour on acceptability

\begin{tabular}{rccccc} 
& $B$ & $S E(B)$ & $B$ & $|t|$ & $p$ \\
\hline Gender & .14 & .21 & .04 & .66 & .510 \\
Age & $<.001$ & .01 & -.002 & -.03 & .974 \\
AUDIT & -.01 & .02 & -.03 & -.31 & .755 \\
Previous Attendance & .26 & .23 & .07 & 1.15 & .25 \\
Alcohol-free Event Attitudes & .46 & .07 & .42 & 6.87 & $<.001^{* *}$ \\
Alcohol Consumption Attitudes & -.15 & .07 & -.18 & -2.03 & $.044^{*}$ \\
Intention & .04 & .07 & .05 & .57 & .572 \\
Subjective Norm & .02 & .08 & .02 & .28 & .784 \\
Perceived behavioural control & .14 & .07 & .11 & 1.94 & .053 \\
Life Satisfaction & .01 & .01 & .04 & .75 & .454 \\
Social Connectedness & -.08 & .08 & -.06 & -1.07 & .286 \\
$\mathrm{R}^{2}$ & .25 & & & & \\
Adjusted $\mathrm{R}^{2}$ & .22 & & & & \\
\hline & & & & &
\end{tabular}

$* \mathrm{p}<.05 \quad * * \mathrm{p}<.01$

Model fit: $F(11,272)=8.089, p<.001$ 
Table 6: Regression analysis examining impact of demographics, alcohol consumption, health cognitions, social connectedness, life satisfaction and past behaviour on support

\begin{tabular}{rccccc} 
& $B$ & $S E(B)$ & $B$ & $|t|$ & $p$ \\
\hline Gender & -.42 & .21 & -.10 & -1.98 & $.049^{*}$ \\
Age & .02 & .01 & .08 & 1.37 & .172 \\
AUDIT & .01 & .02 & .02 & .31 & .758 \\
Previous Attendance & .60 & .23 & .14 & 2.60 & $.010^{* *}$ \\
Alcohol-free Event Attitudes & .64 & .07 & .53 & 9.45 & $<.001^{* *}$ \\
Alcohol Consumption Attitudes & -.11 & .08 & -.12 & -1.48 & .139 \\
Intention & -.12 & .07 & -.16 & -1.86 & .065 \\
Subjective Norm & .11 & .08 & .09 & 1.43 & .153 \\
Perceived behavioural control & .12 & .07 & .08 & 1.56 & .120 \\
Life Satisfaction & .00 & .01 & -.00 & -.01 & .991 \\
Social Connectedness & -.26 & .08 & -.16 & -3.18 & $.002^{* *}$ \\
$\mathrm{R}^{2}$ & .43 & & & & \\
Adjusted $\mathrm{R}^{2}$ & .41 & & & & \\
\hline & & & & &
\end{tabular}

$* \mathrm{p}<.05 \quad * * \mathrm{p}<.01$

Model fit: $F(11,253)=16.799, p<.001$ 